Research Article

\title{
A note on the symmetric division deg coindex of graphs
}

\author{
K. Pattabiraman* \\ Department of Mathematics, Government Arts College (Autonomous), Kumbakonam, India
}

(Received: 6 December 2021. Received in revised form: 22 December 2021. Accepted: 27 December 2021. Published online: 30 December 2021.)

(c) 2021 the author. This is an open access article under the CC BY (International 4.0) license (www.creativecommons.org/licenses/by/4.0/).

\begin{abstract}
The symmetric division deg coindex $\overline{S D D}(G)$ of a simple connected graph $G$ is defined as the sum of the terms $\frac{d_{G}(u)^{2}+d_{G}(v)^{2}}{d_{G}(u) d_{G}(v)}$ over all pairs of distinct non-adjacent vertices of $G$, where $d_{G}(u)$ denotes the degree of a vertex $u$ of $G$. In this paper, upper bounds on the symmetric division deg coindex of edge corona product of two graphs and Mycielskian of a graph are presented. Also, it is proved that the symmetric division deg coindex of the double graph of a connected graph $G$ with $n$ vertices can be written in terms of the symmetric division deg coindex of $G$ and $n$.
\end{abstract}

Keywords: symmetric division deg coindex; edge corona graph; Mycielskian of a graph; double graph.

2020 Mathematics Subject Classification: 05C09, 05 C76.

\section{Introduction}

A topological index of a graph is a parameter that does not depend on the labeling or pictorial representation of the graph. In theoretical chemistry, molecular structure descriptors (also called topological indices) are used for modeling physicochemical, pharmacologic, toxicologic, biological and other properties of chemical compounds. Several types of such indices exist, especially the ones that depend on the vertex and edge distances.

Topological indices have found applications in modeling several physicochemical properties in QSAR (Quantitative structure-activity relationship) and QSPR (quantitative structure-property relationships) studies [4,9]. Many particular types of topological indices are defined using the structure of the underlying molecular graph, such as the Wiener index [11], first Zagreb index [2] and Balaban index [3]. Vukičević and Gašperov [10] observed that most of these indices are defined via the sum of individual bond contributions. Among the 148 discrete Adriatic indices studied in [10], whose predictive properties were evaluated against the benchmark datasets of the International Academy of Mathematical Chemistry, 20 indices were selected as significant predictors of physicochemical properties. One of these useful discrete Adriatic indices is the symmetric division deg index $S D D$ which is defined as

$$
S D D(G)=\sum_{x y \in E(G)}\left(\frac{d_{G}(x)}{d_{G}(y)}+\frac{d_{G}(y)}{d_{G}(x)}\right) .
$$

Among all the existing topological indices, $S D D$ index has the best correlating ability for predicting the total surface area of polychlorobiphenys [10].

The first Zagreb index [2] and its coindex of a connected graph $G$ are defined as

$$
M_{1}(G)=\sum_{u v \in E(G)}\left(d_{G}(u)+d_{G}(v)\right)
$$

and

$$
\bar{M}_{1}(G)=\sum_{u v \notin E(G)}\left(d_{G}(u)+d_{G}(v)\right),
$$

respectively. The harmonic index and its coindex are, respectively, defined as

$$
H(G)=\sum_{u v \in E(G)} \frac{2}{d_{G}(u)+d_{G}(v)}
$$

and

$$
\bar{H}(G)=\sum_{u v \notin E(G)} \frac{2}{d_{G}(u)+d_{G}(v)} .
$$


Motivated by the studies on the coindices, like the first Zagreb coindex and harmonic coindex, the symmetric division deg coindex is proposed here:

$$
\overline{S D D}(G)=\sum_{u v \notin E(G)} \frac{d_{G}(u)^{2}+d_{G}(v)^{2}}{d_{G}(u) d_{G}(v)} .
$$

In this paper, upper bounds on the symmetric division deg coindex of edge corona product of two graphs and Mycielskian of a graph are given. Also, it is proved that the symmetric division deg coindex of the double graph of a connected graph $G$ with $n$ vertices can be written in terms of the symmetric division deg coindex of $G$ and $n$.

\section{Mathematical properties of $\overline{S D D}$}

A modification of the second Zagreb index was proposed by Nikolić et al. [7] in 2003. The modified second Zagreb index and its coindex of $G$ are, respectively, defined as

$$
M_{2}^{*}(G)=\sum_{u v \in E(G)} \frac{1}{d_{G}(u) d_{G}(v)}
$$

and

$$
\bar{M}_{2}^{*}(G)=\sum_{u v \notin E(G)} \frac{1}{d_{G}(u) d_{G}(v)} .
$$

The inverse degree index of $G$ is defined as

$$
I D(G)=\sum_{u \in V(G)} \frac{1}{d_{G}(u)} .
$$

The redefined first and second Zagreb coindices of $G$ are, respectively, defined as

$$
\overline{R Z}_{1}(G)=\sum_{u v \notin E(G)} \frac{d_{G}(u)+d_{G}(v)}{d_{G}(u) d_{G}(v)}
$$

and

$$
\overline{R Z}_{2}(G)=\sum_{u v \notin E(G)} \frac{d_{G}(u)^{2}+d_{G}(v)^{2}}{d_{G}(u)+d_{G}(v)} .
$$

In this section, we present upper bounds on the symmetric division deg coindex of edge corona product graph, Mycielskian of a graph and double graphs.

The edge corona product $G \bullet H$ of $G$ and $H$ is defined as the graph obtained by taking one copy of $G$ and $|E(G)|$ copies of $H$, and then joining two end vertices of the $i^{t h}$ edge of $G$ to every vertex in the $i^{\text {th }}$ copy of $H$ (see [1,3,8] for more details).

Lemma 2.1. [6] Let $f$ be a convex function on the interval I and $x_{1}, x_{2}, \ldots, x_{n} \in I$. Then

$$
f\left(\frac{x_{1}+x_{2}+\ldots+x_{n}}{n}\right) \leq \frac{f\left(x_{1}\right)+f\left(x_{2}\right)+\ldots f\left(x_{n}\right)}{n},
$$

with equality if and only if $x_{1}=x_{2}=\ldots=x_{n}$.

Theorem 2.1. Let $G_{1}$ and $G_{2}$ be two graphs with $n_{1}, n_{2}$ vertices and $m_{1}, m_{2}$ edges, respectively. Then

$$
\begin{aligned}
\overline{S D D}\left(G_{1} \bullet G_{2}\right) \leq & \overline{S D D}\left(G_{1}\right)+m_{1}\left(\overline{S D D}\left(G_{2}\right)+\overline{R Z_{1}}\left(G_{2}\right)+8 \overline{M_{2}^{*}}\left(G_{2}\right)+\overline{R Z_{2}}\left(G_{2}\right)+\bar{H}\left(G_{2}\right)\right)+\frac{2 n_{1}\left(n_{2}+m_{2}\right)}{\left(n_{2}+1\right)}\left(I D\left(G_{1}\right)-1\right) \\
& +\left(\left(n_{2}+1\right)\left(2 n_{1} m_{1}-M_{1}\left(G_{1}\right)\right)+2 m_{1}\left(m_{1}-1\right)\left(m_{2}+1\right)\right) I D\left(G_{2}\right)+2 m_{1}\left(m_{2}^{2}-m_{2}-2 n_{2}\right) .
\end{aligned}
$$

Proof. Let $x_{i j}$ be the $j$ th vertex in the $i$ th copy of $H, i \in\left\{1,2, \ldots, m_{1}\right\}, j \in\left\{1,2, \ldots, n_{2}\right\}$, and let $y_{k}$ be the $k$ th vertex in $G_{1}$, $k \in\left\{1,2, \ldots, n_{1}\right\}$. Also let $x_{j}$ be the $j$ th vertex in $G_{2}$. By the definition of edge corona of $G_{1}$ and $G_{2}$, for each vertex $x_{i j}$, we have $d_{G_{1}} \bullet G_{2}\left(x_{i j}\right)=d_{G_{2}}\left(x_{j}\right)+2$, and for every vertex $y_{k}$ in $G_{1}, d_{G_{1}} \bullet G_{2}\left(y_{k}\right)=d_{G_{1}}\left(y_{k}\right) n_{2}+d_{G_{1}}\left(y_{k}\right)=\left(n_{2}+1\right) d_{G_{1}}\left(y_{k}\right)$.

Now, we consider the following four cases of nonadjacent vertex pairs in $G_{1} \bullet G_{2}$.

Case 1. The nonadjacent vertex pairs $\left\{x_{i j}, x_{i h}\right\}, 1 \leq i \leq m_{1}, 1 \leq j<h \leq n_{2}$, are considered and it is assumed that $x_{j} x_{h} \notin E\left(G_{2}\right)$.

$$
S_{1}=\sum_{i=1}^{m_{1}} \sum_{x_{i j} x_{i h} \notin E\left(G_{1} \bullet G_{2}\right)} \frac{d_{G_{1}} \bullet G_{2}\left(x_{i j}\right)^{2}+d_{G_{1}} \bullet G_{2}\left(x_{i h}\right)^{2}}{d_{G_{1} \bullet G_{2}}\left(x_{i j}\right) d_{G_{1} \bullet G_{2}}\left(x_{i h}\right)}
$$




$$
\begin{aligned}
& =\sum_{i=1}^{m_{1}} \sum_{x_{j} x_{h} \notin E\left(G_{2}\right)} \frac{\left(d_{G_{2}}\left(x_{j}\right)+2\right)^{2}+\left(d_{G_{2}}\left(x_{h}\right)+2\right)^{2}}{\left(d_{G_{2}}\left(x_{j}\right)+2\right)\left(d_{G_{2}}\left(x_{h}\right)+2\right)} \\
& =\sum_{i=1}^{m_{1}} \sum_{x_{j} x_{h} \notin E\left(G_{2}\right)} \frac{\left(d_{G_{2}}\left(x_{j}\right)^{2}+d_{G_{2}}\left(x_{h}\right)^{2}\right)+4\left(d_{G_{2}}\left(x_{h}\right)+d_{G_{2}}\left(x_{j}\right)\right)+8}{d_{G_{2}}\left(x_{j}\right) d_{G_{2}}\left(x_{h}\right)+2\left(d_{G_{2}}\left(x_{j}\right)+d_{G_{2}}\left(x_{h}\right)\right)+4} .
\end{aligned}
$$

By Lemma 2.1, we have

$$
\frac{1}{d_{G_{2}}\left(x_{j}\right) d_{G_{2}}\left(x_{h}\right)+2\left(d_{G_{2}}\left(x_{j}\right)+d_{G_{2}}\left(x_{h}\right)\right)+4} \leq \frac{1}{4 d_{G_{2}}\left(x_{j}\right) d_{G_{2}}\left(x_{h}\right)}+\frac{1}{8\left(d_{G_{2}}\left(x_{j}\right)+d_{G_{2}}\left(x_{h}\right)\right)+16}
$$

with equality if and only if $d_{G_{2}}\left(x_{j}\right) d_{G_{2}}\left(x_{h}\right)=8\left(d_{G_{2}}\left(x_{j}\right)+d_{G_{2}}\left(x_{h}\right)\right)+16$. Thus,

$$
\begin{aligned}
S_{1} \leq & \sum_{i=1}^{m_{1}} \sum_{x_{j} x_{h} \notin E\left(G_{2}\right)}\left(\frac{\left(d_{G_{2}}\left(x_{j}\right)^{2}+d_{G_{2}}\left(x_{h}\right)^{2}\right)+4\left(d_{G_{2}}\left(x_{h}\right)+d_{G_{2}}\left(x_{j}\right)\right)+8}{4 d_{G_{2}}\left(x_{j}\right) d_{G_{2}}\left(x_{h}\right)}\right) \\
& \left.+\sum_{i=1}^{m_{1}} \sum_{x_{j} x_{h} \notin E\left(G_{2}\right)} \frac{\left(d_{G_{2}}\left(x_{j}\right)^{2}+d_{G_{2}}\left(x_{h}\right)^{2}\right)+4\left(d_{G_{2}}\left(x_{h}\right)+d_{G_{2}}\left(x_{j}\right)\right)+8}{8\left(d_{G_{2}}\left(x_{j}\right)+d_{G_{2}}\left(x_{h}\right)\right)+16}\right) .
\end{aligned}
$$

Note that $8\left(d_{G_{2}}\left(x_{j}\right)+d_{G_{2}}\left(x_{h}\right)\right)+16 \geq d_{G_{2}}\left(x_{j}\right)+d_{G_{2}}\left(x_{h}\right)$. This implies

$$
\frac{1}{8\left(d_{G_{2}}\left(x_{j}\right)+d_{G_{2}}\left(x_{h}\right)\right)+16} \leq \frac{1}{d_{G_{2}}\left(x_{j}\right)+d_{G_{2}}\left(x_{h}\right)} .
$$

Therefore,

$$
\begin{aligned}
S_{1} \leq & \sum_{i=1}^{m_{1}} \sum_{x_{j} x_{h} \notin E\left(G_{2}\right)}\left(\frac{\left(d_{G_{2}}\left(x_{j}\right)^{2}+d_{G_{2}}\left(x_{h}\right)^{2}\right)+4\left(d_{G_{2}}\left(x_{h}\right)+d_{G_{2}}\left(x_{j}\right)\right)+8}{d_{G_{2}}\left(x_{j}\right) d_{G_{2}}\left(x_{h}\right)}\right. \\
& \left.+\sum_{i=1}^{m_{1}} \sum_{x_{j} x_{h} \notin E\left(G_{2}\right)} \frac{\left(d_{G_{2}}\left(x_{j}\right)^{2}+d_{G_{2}}\left(x_{h}\right)^{2}\right)+4\left(d_{G_{2}}\left(x_{h}\right)+d_{G_{2}}\left(x_{j}\right)\right)+8}{d_{G_{2}}\left(x_{j}\right)+d_{G_{2}}\left(x_{h}\right)}\right) \\
= & m_{1}\left(\overline{S D D}\left(G_{2}\right)+\overline{R Z_{1}}\left(G_{2}\right)+8 \overline{M_{2}}\left(G_{2}\right)+\overline{R Z_{2}}\left(G_{2}\right)+\bar{H}\left(G_{2}\right)+4 \overline{m_{2}}\right) .
\end{aligned}
$$

Case 2. The nonadjacent vertex pairs $\left\{y_{k}, y_{s}\right\}, 1 \leq k<s \leq n_{1}$, are considered and it is assumed that $y_{k} y_{s} \notin E\left(G_{1}\right)$. Thus,

$$
\begin{aligned}
S_{2} & =\sum_{y_{k} y_{s} \notin E\left(G_{1} \bullet G_{2}\right)} \frac{d_{G_{1} \bullet G_{2}}\left(y_{k}\right)^{2}+d_{G_{1} \bullet G_{2}}\left(y_{s}\right)^{2}}{d_{G_{1} \bullet G_{2}}\left(y_{k}\right) d_{G_{1} \bullet G_{2}}\left(y_{s}\right)} \\
& =\sum_{y_{k} y_{s} \notin E\left(G_{1}\right)} \frac{\left(n_{2}+1\right)^{2} d_{G_{1}}\left(y_{k}\right)^{2}+\left(n_{2}+1\right)^{2} d_{G_{1}}\left(y_{s}\right)^{2}}{\left(n_{2}+1\right)^{2} d_{G_{1}}\left(y_{k}\right) d_{G_{1}}\left(y_{s}\right)} \\
& =\sum_{y_{k} y_{s} \notin E\left(G_{1}\right)} \frac{d_{G_{1}}\left(y_{k}\right)^{2}+d_{G_{1}}\left(y_{s}\right)^{2}}{d_{G_{1}}\left(y_{k}\right) d_{G_{1}}\left(y_{s}\right)} \\
& =\overline{S D D}\left(G_{1}\right) .
\end{aligned}
$$

Case 3. The nonadjacent vertex pairs $\left\{x_{i j}, y_{k}\right\}, 1 \leq i \leq m_{1}, 1 \leq j \leq n_{2}, 1 \leq k \leq n_{1}$, are considered and it is assumed that the $i$ th edge $e_{i}, 1 \leq i \leq m_{1}$, in $G_{1}$ does not pass through $y_{k}$. Note that each vertex $y_{k}$ is adjacent to all vertices of $d_{G_{1}}\left(y_{k}\right)$ copies of $G_{2}$, that is, each $y_{k}$ is not adjacent to any vertex of $m_{1}-d_{G_{1}}\left(y_{k}\right)$ copies of $G_{2}$. Hence,

$$
\begin{aligned}
S_{3} & =\sum_{k=1}^{n_{1}}\left(n_{1}-d_{G_{1}}\left(y_{k}\right)\right) \sum_{j=1}^{n_{2}} \frac{\left(d_{G_{2}}\left(x_{j}\right)+2\right)^{2}+\left(n_{2}+1\right)^{2} d_{G_{1}}\left(y_{k}\right)^{2}}{\left(d_{G_{2}}\left(x_{j}\right)+2\right)\left(n_{2}+1\right) d_{G_{1}}\left(y_{k}\right)} \\
& \leq \sum_{k=1}^{n_{1}}\left(n_{1}-d_{G_{1}}\left(y_{k}\right)\right) \sum_{j=1}^{n_{2}}\left(\frac{\left(d_{G_{2}}\left(x_{j}\right)+2\right)}{\left(n_{2}+1\right) d_{G_{1}}\left(y_{k}\right)}+\frac{\left(n_{2}+1\right) d_{G_{1}}\left(y_{k}\right)}{d_{G_{2}}\left(x_{j}\right)}\right) \\
& =\sum_{k=1}^{n_{1}}\left(n_{1}-d_{G_{1}}\left(y_{k}\right)\right)\left(\frac{2\left(n_{2}+m_{2}\right)}{\left(n_{2}+1\right) d_{G_{1}}\left(y_{k}\right)}+\left(n_{2}+1\right) d_{G_{1}}\left(y_{k}\right) I D\left(G_{2}\right)\right) \\
& =\frac{2 n_{1}\left(n_{2}+m_{2}\right)}{\left(n_{2}+1\right)} I D\left(G_{1}\right)+2 n_{1} m_{1}\left(n_{2}+1\right) I D\left(G_{2}\right)-\frac{2 n_{1}\left(n_{2}+m_{2}\right)}{\left(n_{2}+1\right)}-\left(n_{2}+1\right) M_{1}\left(G_{1}\right) I D\left(G_{2}\right)
\end{aligned}
$$




$$
=\frac{2 n_{1}\left(n_{2}+m_{2}\right)}{\left(n_{2}+1\right)}\left(I D\left(G_{1}\right)-1\right)+\left(n_{2}+1\right)\left(2 n_{1} m_{1}-M_{1}\left(G_{1}\right)\right) I D\left(G_{2}\right) .
$$

Case 4. The nonadjacent vertex pairs $\left\{x_{i j}, x_{\ell h}\right\}, 1 \leq i<\ell \leq m_{1}, 1 \leq j, h \leq n_{2}$, are considered.

$$
\begin{aligned}
S_{4} & =\sum_{x_{i j} x_{\ell h} \notin E\left(G_{1} \bullet G_{2}\right)} \frac{d_{G_{1} \bullet G_{2}}\left(x_{i j}\right)^{2}+d_{G_{1} \bullet G_{2}}\left(x_{\ell h}\right)^{2}}{d_{G_{1} \bullet G_{2}}\left(x_{i j}\right) d_{G_{1} \bullet G_{2}}\left(x_{\ell h}\right)} \\
& =\frac{m_{1}\left(m_{1}-1\right)}{2} \sum_{j=1}^{n_{2}} \sum_{h=1}^{n_{2}} \frac{\left(d_{G_{2}}\left(x_{j}\right)+2\right)^{2}+\left(d_{G_{2}}\left(x_{h}\right)+2\right)^{2}}{\left(d_{G_{2}}\left(x_{j}\right)+2\right)\left(d_{G_{2}}\left(x_{h}\right)+2\right)} \\
& \leq \frac{m_{1}\left(m_{1}-1\right)}{2} \sum_{j=1}^{n_{2}} \sum_{h=1}^{n_{2}}\left(\frac{\left(d_{G_{2}}\left(x_{j}\right)+2\right)}{d_{G_{2}}\left(x_{h}\right)}+\frac{\left(d_{G_{2}}\left(x_{h}\right)+2\right)}{d_{G_{2}}\left(x_{j}\right)}\right) \\
& =2 m_{1}\left(m_{1}-1\right)\left(m_{2}+1\right) I D\left(G_{2}\right) .
\end{aligned}
$$

From the above four cases of nonadjacent vertex pairs, one obtains the desired result.

The Mycielskian $\mu(G)$ (see [5]) of $G$ contains $G$ itself as an isomorphic subgraph, together with $n+1$ additional vertices: a vertex $u_{i}$ corresponding to each vertex $v_{i}$ of $G$, and another vertex $w$. Each vertex $u_{i}$ is connected by an edge to $w$, so that these vertices form a subgraph in the form of a star $K_{1, n}$. The minimum and maximum vertex degrees of $G$, respectively, are denoted by $\delta(G)$ and $\Delta(G)$.

By the definition of the Mycielskian of a graph $G$, for each edge $v_{i} v_{j}$ of $G$, the Mycielskian of $G$ includes two edges, $u_{i} v_{j}$ and $v_{i} u_{j}$. Now, we find an upper bound for symmetric division deg coindex of the Mycielskian of a graph.

Theorem 2.2. Let $G$ be a graph on $n$ vertices and medges. Then

$$
\begin{aligned}
\overline{S D D}(\mu(G)) \leq & \left(\frac{n(n-1)-2(m-1)}{2}\right) \overline{S D D}(G)+\left(\frac{n(n-1)-2 m)}{2}\right)\left(2 \overline{R Z}_{1}(G)+\bar{M}_{2}^{*}(G)+\overline{R Z}_{2}(G)+\bar{H}(G)+n(n-1)-m\right) \\
& +m\left(S D D(G)+M_{2}^{*}(G)+R Z_{2}(G)+H(G)+2(m+n)\right)+\left(n^{2}+2 n+2\right) I D(G)+(3 n+8 m)+\frac{3 \Delta^{2}+2 \Delta+1}{\delta^{2}}
\end{aligned}
$$

Proof. Let $V(G)=\left\{v_{1}, \ldots, v_{n}\right\}$ and let $V(\mu(G))=\left\{v_{1}, \ldots, v_{n}, u_{1}, \ldots, u_{n}, w\right\}$. By the structure of the Mycielskian of $G$, if $v_{i} v_{j} \notin E(G)$, then $v_{i} u_{j} \notin E(G)$, and $v_{j} u_{i} \notin E(G)$. By the definition of $\mu(G)$, for each $i \in\{1,2, \ldots, n\}$, we have $d_{\mu(G)}\left(v_{i}\right)=$ $2 d_{G}\left(v_{i}\right), d_{\mu(G)}\left(u_{i}\right)=d_{G}\left(v_{i}\right)+1$ and $d_{\mu(G)}(w)=n$. Now, we consider the following cases of nonadjacent vertex pairs in $\mu(G)$.

Case 1. The nonadjacent vertex pairs $\left\{v_{i}, v_{j}\right\}$ in $\mu(G)$ are considered.

$$
C_{1}=\sum_{v_{i} v_{j} \notin E(\mu(G))} \frac{d_{\mu(G)}\left(v_{i}\right)^{2}+d_{\mu(G)}\left(v_{j}\right)^{2}}{d_{\mu(G)}\left(v_{i}\right) d_{\mu(G)}\left(v_{j}\right)}=\sum_{v_{i} v_{j} \notin E(G)} \frac{4 d_{G}\left(v_{i}\right)^{2}+4 d_{G}\left(v_{j}\right)^{2}}{4 d_{G}\left(v_{i}\right) d_{G}\left(v_{j}\right)}=\overline{S D D}(G) .
$$

Case 2. The nonadjacent vertex pairs $\left\{u_{i}, u_{j}\right\}$ in $\mu(G)$ are considered.

Case 2.1. $u_{i} u_{j} \notin E(\mu(G))$ and $v_{i} v_{j} \notin E(G)$.

$$
\begin{aligned}
C_{2}^{\prime} & =\sum_{u_{i} u_{j} \notin E(\mu(G))} \frac{d_{\mu(G)}\left(u_{i}\right)^{2}+d_{\mu(G)}\left(u_{j}\right)^{2}}{d_{\mu(G)}\left(u_{i}\right) d_{\mu(G)}\left(u_{j}\right)} \\
& =\sum_{v_{i} v_{j} \notin E(G)} \frac{\left(d_{G}\left(v_{i}\right)+1\right)^{2}+\left(d_{G}\left(v_{j}\right)+1\right)^{2}}{\left(d_{G}\left(v_{i}\right)+1\right)\left(d_{G}\left(v_{j}\right)+1\right)} \\
& =\sum_{v_{i} v_{j} \notin E(G)} \frac{\left(d_{G}\left(v_{i}\right)^{2}+d_{G}\left(v_{j}\right)^{2}\right)+2\left(d_{G}\left(v_{i}\right)+d_{G}\left(v_{j}\right)\right)+2}{d_{G}\left(v_{i}\right) d_{G}\left(v_{j}\right)+\left(d_{G}\left(v_{i}\right)+d_{G}\left(v_{j}\right)\right)+1} .
\end{aligned}
$$

By Lemma 2.1, one obtains

$$
\begin{aligned}
C_{2}^{\prime} \leq & \sum_{v_{i} v_{j} \notin E(G)} \frac{\left(d_{G}\left(v_{i}\right)^{2}+d_{G}\left(v_{j}\right)^{2}\right)+2\left(d_{G}\left(v_{i}\right)+d_{G}\left(v_{j}\right)\right)+2}{4 d_{G}\left(v_{i}\right) d_{G}\left(v_{j}\right)} \\
& +\sum_{v_{i} v_{j} \notin E(G)} \frac{\left(d_{G}\left(v_{i}\right)^{2}+d_{G}\left(v_{j}\right)^{2}\right)+2\left(d_{G}\left(v_{i}\right)+d_{G}\left(v_{j}\right)\right)+2}{\left.4\left(d_{G}\left(v_{i}\right)+d_{G}\left(v_{j}\right)\right)+1\right)}
\end{aligned}
$$




$$
\begin{aligned}
\leq & \sum_{v_{i} v_{j} \notin E(G)} \frac{\left(d_{G}\left(v_{i}\right)^{2}+d_{G}\left(v_{j}\right)^{2}\right)+2\left(d_{G}\left(v_{i}\right)+d_{G}\left(v_{j}\right)\right)+2}{d_{G}\left(v_{i}\right) d_{G}\left(v_{j}\right)} \\
& +\sum_{v_{i} v_{j} \notin E(G)} \frac{\left(d_{G}\left(v_{i}\right)^{2}+d_{G}\left(v_{j}\right)^{2}\right)+2\left(d_{G}\left(v_{i}\right)+d_{G}\left(v_{j}\right)\right)+2}{d_{G}\left(v_{i}\right)+d_{G}\left(v_{j}\right)} \\
= & \overline{S D D}(G)+2 \overline{R Z}_{1}(G)+\bar{M}_{2}^{*}(G)+\overline{R Z}_{2}(G)+\bar{H}(G)+n(n-1)-m .
\end{aligned}
$$

Case 2.2. $u_{i} u_{j} \notin E(\mu(G))$ and $v_{i} v_{j} \in E(G)$.

$$
\begin{aligned}
C_{2}^{\prime \prime} & =\sum_{u_{i} u_{j} \notin E(\mu(G))} \frac{d_{\mu(G)}\left(u_{i}\right)^{2}+d_{\mu(G)}\left(u_{j}\right)^{2}}{d_{\mu(G)}\left(u_{i}\right) d_{\mu(G)}\left(u_{j}\right)} \\
& =\sum_{v_{i} v_{j} \in E(G)} \frac{\left(d_{G}\left(v_{i}\right)+1\right)^{2}+\left(d_{G}\left(v_{j}\right)+1\right)^{2}}{\left(d_{G}\left(v_{i}\right)+1\right)\left(d_{G}\left(v_{j}\right)+1\right)} \\
& \leq S D D(G)+M_{2}^{*}(G)+R Z_{2}(G)+H(G)+2(m+n) .
\end{aligned}
$$

If $u_{i} u_{j} \notin E(\mu(G))$, then there are $m$ edges $v_{i} v_{j} \in E(G)$ and $\frac{n(n-1)}{2}-m$ nonadjacent vertex pairs $\left\{v_{i}, v_{j}\right\}$ in $G$ as well as $\mu(G)$. By Cases 2.1 and 2.2, we have the contribution of nonadjacent vertex pair of Case 2 given as

$$
\begin{aligned}
C_{2}= & \left(\frac{n(n-1)}{2}-m\right) C_{2}^{\prime}+m C_{2}^{\prime \prime} \\
\leq & \left(\frac{n(n-1)}{2}-m\right)\left(\overline{S D D}(G)+2 \overline{R Z}_{1}(G)+\bar{M}_{2}^{*}(G)+\overline{R Z}_{2}(G)+\bar{H}(G)+n(n-1)-m\right) \\
& +m\left(S D D(G)+M_{2}^{*}(G)+R Z_{2}(G)+H(G)+2(m+n)\right) .
\end{aligned}
$$

Case 3. The nonadjacent vertex pairs $\left\{u_{i}, v_{i}\right\}$ in $\mu(G)$ are considered for each $i=1,2, \ldots, n$.

$$
\begin{aligned}
C_{3} & =\sum_{i=1}^{n} \frac{d_{\mu(G)}\left(u_{i}\right)^{2}+d_{\mu(G)}\left(v_{i}\right)^{2}}{d_{\mu(G)}\left(u_{i}\right) d_{\mu(G)}\left(v_{i}\right)} \\
& =\sum_{i=1}^{n} \frac{\left(d_{G}\left(v_{i}\right)+1\right)^{2}+4 d_{G}\left(v_{i}\right)^{2}}{2\left(d_{G}\left(v_{i}\right)+1\right)\left(d_{G}\left(v_{i}\right)\right)} \\
& \leq \sum_{i=1}^{n}\left(\frac{d_{G}\left(v_{i}\right)+1}{d_{G}\left(v_{i}\right)}+2\right) .
\end{aligned}
$$

Thus, $C_{3} \leq I D(G)+3 n$.

Case 4. The nonadjacent vertex pairs $\left\{u_{i}, v_{j}\right\}$ in $\mu(G)$ are considered.

$$
\begin{aligned}
C_{4} & =\sum_{u_{i} v_{j} \notin E(\mu(G))} \frac{d_{\mu(G)}\left(u_{i}\right)^{2}+d_{\mu(G)}\left(v_{j}\right)^{2}}{d_{\mu(G)}\left(u_{i}\right) d_{\mu(G)}\left(v_{j}\right)} \\
& =\sum_{v_{i} v_{j} \notin E(G)} \frac{\left(d_{G}\left(v_{i}\right)+1\right)^{2}+4 d_{G}\left(v_{j}\right)^{2}}{2\left(d_{G}\left(v_{i}\right)+1\right) d_{G}\left(v_{j}\right)} .
\end{aligned}
$$

For any vertex $x \in V(G)$, one has $\delta(G) \leq d_{G}(x) \leq \Delta(G)$ and hence

$$
\begin{aligned}
C_{4} & \leq \sum_{v_{i} v_{j} \notin E(G)} \frac{\left(d_{G}\left(v_{i}\right)+1\right)^{2}+4 d_{G}\left(v_{j}\right)^{2}}{d_{G}\left(v_{i}\right) d_{G}\left(v_{j}\right)} \\
& \leq \overline{S D D}(G)+\frac{3 \Delta^{2}+2 \Delta+1}{\delta^{2}} .
\end{aligned}
$$

Case 5. The nonadjacent vertex pairs $\left\{w, v_{i}\right\}$ in $\mu(G)$ are considered for each $i=1,2, \ldots, n$.

$$
\begin{aligned}
C_{5} & =\sum_{v_{i} w \notin E(\mu(G))} \frac{d_{\mu(G)}\left(v_{i}\right)^{2}+d_{\mu(G)}(w)^{2}}{d_{\mu(G)}\left(v_{i}\right) d_{\mu(G)}(w)} \\
& =\sum_{v_{i} \in V(G)} \frac{4 d_{G}\left(v_{i}\right)^{2}+(n+1)^{2}}{2 d_{G}\left(v_{i}\right)(n+1)}
\end{aligned}
$$




$$
\begin{aligned}
& \leq \sum_{v_{i} \in V(G)} \frac{4 d_{G}\left(v_{i}\right)^{2}+(n+1)^{2}}{d_{G}\left(v_{i}\right)} \\
& =(n+1)^{2} I D(G)+8 m .
\end{aligned}
$$

From the above five cases of nonadjacent vertex pairs, one obtains the desired result.

Let $G$ be a graph with $V(G)=\left\{v_{1}, v_{2}, \ldots, v_{n}\right\}$. The vertices of the double graph $G^{*}$ are given by the two sets $X=$ $\left\{x_{1}, x_{2}, \ldots, x_{n}\right\}$ and $Y=\left\{y_{1}, y_{2}, \ldots, y_{n}\right\}$. Thus for each vertex $v_{i} \in V(G)$, there are two vertices $x_{i}$ and $y_{i}$ in $V\left(G^{*}\right)$. The double graph $G^{*}$ includes the initial edge set of each copy of $G$, and for any edge $v_{i} v_{j} \in E(G)$, two more edges $x_{i} y_{j}$ and $x_{j} y_{i}$ are added. For a given vertex $v$ in $G$, let

$$
T_{G}(v)=\sum_{u v \notin E(G)} \frac{d_{G}(u)^{2}+d_{G}(v)^{2}}{d_{G}(u) d_{G}(v)} .
$$

Now, we find the exact value of the symmetric division deg coindex for the double graph of a given graph.

Theorem 2.3. Let $G$ be a connected graph with $n$ vertices. Then $\overline{S D D}\left(G^{*}\right)=4 \overline{S D D}(G)+2 n$.

Proof. Let $V(G)=\left\{v_{1}, v_{2}, \ldots, v_{n}\right\}$. Suppose that $x_{i}$ and $y_{i}$ are the corresponding clone vertices, in $G^{*}$, of $v_{i}$ for each $i \in$ $\{1,2, \ldots, n\}$. For any given vertex $v_{i}$ in $G$ and its clone vertices $x_{i}$ and $y_{i}, d_{G^{*}}\left(x_{i}\right)=d_{G^{*}}\left(y_{i}\right)=2 d_{G}\left(v_{i}\right)$ by the definition of the double graph. For $v_{i}, v_{j} \in V(G)$, if $v_{i} v_{j} \notin E(G)$, then $x_{i} x_{j} \notin E(G), y_{i} y_{j} \notin E(G), x_{i} y_{j} \notin E(G)$ and $y_{i} x_{j} \notin E(G)$. Hence, we only consider total contribution of the following three types of nonadjacent vertex pairs to calculate $\overline{S D D}(G)$.

Case 1. The nonadjacent vertex pairs $\left\{x_{i}, x_{j}\right\}$ and $\left\{y_{i}, y_{j}\right\}$ are considered, where $v_{i} v_{j} \notin E(G)$.

$$
\begin{aligned}
\sum_{y_{i} y_{j} \notin E\left(G^{*}\right)} \frac{d_{G^{*}}\left(y_{i}\right)^{2}+d_{G^{*}}\left(y_{j}\right)^{2}}{d_{G^{*}}\left(y_{i}\right) d_{G^{*}}\left(y_{j}\right)} & =\sum_{x_{i} x_{j} \notin E\left(G^{*}\right)} \frac{d_{G^{*}}\left(x_{i}\right)^{2}+d_{G^{*}}\left(x_{j}\right)^{2}}{d_{G^{*}}\left(x_{i}\right) d_{G^{*}}\left(x_{j}\right)} \\
& =\sum_{v_{i} v_{j} \notin E(G)} \frac{\left.4 d_{G}\left(v_{i}\right)^{2}+4 d_{G} v_{j}\right)^{2}}{\left.4 d_{G}\left(v_{i}\right) d_{G} v_{j}\right)} \\
& =\overline{S D D}(G) .
\end{aligned}
$$

Case 2. The nonadjacent vertex pairs $\left\{x_{i}, y_{i}\right\}$ are considered for each $i \in\{1,2, \ldots, n\}$.

$$
\sum_{i=1}^{n} \frac{d_{G^{*}}\left(x_{i}\right)^{2}+d_{G^{*}}\left(y_{i}\right)^{2}}{d_{G^{*}}\left(x_{i}\right) d_{G^{*}}\left(y_{i}\right)}=\sum_{i=1}^{n} \frac{4 d_{G}\left(v_{i}\right)^{2}+4 d_{G}\left(v_{i}\right)^{2}}{4 d_{G}\left(v_{i}\right)^{2}}=2 n .
$$

Case 3. The nonadjacent vertex pairs $\left\{x_{i}, y_{j}\right\}$ and $\left\{y_{i}, x_{j}\right\}$ are considered, where $v_{i} v_{j} \notin E(G)$.

For each $x_{i}$, there exist $n-1-d_{G}\left(v_{i}\right)$ vertices in the set $\left\{y_{1}, y_{2}, \ldots, y_{n}\right\}$, among which every vertex together with $x_{i}$ compose a nonadjacent vertex pairs of $G^{*}$. The total contribution of these $n-1-d_{G}\left(v_{i}\right)$ nonadjacent vertex pairs to calculate $\overline{S D D}\left(G^{*}\right)$ is

$$
\sum_{x_{i} y_{j} \notin E\left(G^{*}\right)} \frac{d_{G^{*}}\left(x_{i}\right)^{2}+d_{G^{*}}\left(y_{j}\right)^{2}}{d_{G^{*}}\left(x_{i}\right) d_{G^{*}}\left(y_{j}\right)}=\sum_{v_{i} v_{j} \notin E\left(G^{*}\right)} \frac{4 d_{G}\left(v_{i}\right)^{2}+4 d_{G}\left(v_{j}\right)^{2}}{4 d_{G}\left(v_{i}\right) d_{G}\left(v_{j}\right)}=T_{G}\left(v_{i}\right) .
$$

Hence,

$$
\sum_{i \neq j, x_{i} y_{j} \notin E\left(G^{*}\right)} \frac{d_{G^{*}}\left(x_{i}\right) 2+d_{G^{*}}\left(y_{j}\right)^{2}}{d_{G^{*}}\left(x_{i}\right) d_{G^{*}}\left(y_{j}\right)}=\sum_{i=1}^{n} T_{G}\left(v_{i}\right)=2 \overline{S D D}(G) .
$$

Therefore,

$$
\begin{aligned}
\overline{S D D}\left(G^{*}\right)= & \sum_{x_{i} x_{j} \notin E\left(G^{*}\right)} \frac{d_{G^{*}}\left(x_{i}\right)^{2}+d_{G^{*}}\left(x_{j}\right)^{2}}{d_{G^{*}}\left(x_{i}\right) d_{G^{*}}\left(x_{j}\right)}+\sum_{y_{i} y_{j} \notin E\left(G^{*}\right)} \frac{d_{G^{*}}\left(y_{i}\right)^{2}+d_{G^{*}}\left(y_{j}\right)^{2}}{d_{G^{*}}\left(y_{i}\right) d_{G^{*}}\left(y_{j}\right)} \\
& +\sum_{i=1}^{n} \frac{d_{G^{*}}\left(x_{i}\right) 2+d_{G^{*}}\left(y_{i}\right)^{2}}{d_{G^{*}}\left(x_{i}\right) d_{G^{*}}\left(y_{i}\right)}+\sum_{i \neq j, x_{i} y_{j} \notin E\left(G^{*}\right)} \frac{d_{G^{*}}\left(x_{i}\right)^{2}+d_{G^{*}}\left(y_{j}\right)^{2}}{d_{G^{*}}\left(x_{i}\right) d_{G^{*}}\left(y_{j}\right)} \\
= & 4 \overline{S D D}(G)+2 n .
\end{aligned}
$$




\section{References}

[1] I. R. Abdolhosseinzadeh, F. Rahbarnia, M. Tavakoli, A. R. Ashrafi, Some vertex-degree-based topological indices under edge corona product, Ital. J. Pure Appl. Math. 38 (2017) 81-91.

[2] I. Gutman, N. Trinajstić, Graph theory and molecular orbitals. Total $\pi$-electron energy of alternant hydrocarbons, Chem. Phys. Lett. 17 (1972) $535-538$.

[3] Y. Hou, W. C. Shiu, The spectrum of the edge corona of two graphs, Electron. J. Linear Algebra 20 (2010) 586-594.

[4] M. Karelson, Molecular Descriptors in QSAR/QSPR, Wiley-Interscience, New York, 2000.

[5] J. Mycielski, Sur le colouring des graphes, Colloq. Math. 3 (1955) 161-162.

[6] C. Niculescu, L. E. Persson, Convex Functions and Their Applications: A Contemporary Approach, Springer, New York, 2006.

[7] S. Nikolić, G. Kovačević, A. Miličević, N. Trinajstić, The Zagreb indices 30 years after, Croat. Chem. Acta 76 (2003) $113-124$.

[8] R. Rinurwati, S. Slamin, H. Suprajitno, General results of local metric dimensions of edge-corona of graphs, Int. Math. Forum 11 (2016) 793-799.

[9] R. Todeschini, V. Consonni, Handbook of Molecular Descriptors, Wiley-VCH, Weinheim, 2000.

[10] D. Vukičević, M. Gašperov, Bond aditive mdelling 1. Ariatic indices, Croat. Chem. Acta 83 (2010) 243-260.

[11] H. Wiener, Structural determination of paraffin boiling points, J. Amer. Chem. Soc. 69 (1947) 17-20. 